Tast, David

[45]

\section{Corning introduces a new technology for mass production of microarrays}

\author{
David Tast, Vic Myer, Mary Trounstine \& Laurent Picard
}

Corning Inc., Corning New York 14831, USA

Corning has developed a new manufacturing process that enables high-speed, high-capacity production of microarrays. Through a unique combination of technologies, thousands of spots are deposited simultaneously, in a massively parallel manner and at high density, onto specially formulated glass slides. The process and the materials used inherently deliver a high level of array reproducibility, in terms of both physical attributes (spot size, spot volume and grid alignment) and performance attributes (sensitivity, dynamic range and low coefficients of variation). We will present data demonstrating the performance of arrays printed with human expressed sequence tags, and we will describe the process in detail.

Tonko, Martin

\section{Gene expression profiling of leukemia cells} during glucocorticoid-induced apoptosis

Martin Tonko, Roman Crazzolarar, Anita Kofler, David Bernhard, Albert Amberger, Bernhard Meister $\&$ Reinhard Kofler

Tyrolean Cancer Research Institute, Innrain 66, 6020 Innsbruck, Austria

Glucocorticoids induce apoptosis in cells of the lymphoid lineage and constitute a central component in the therapy of malignant lymphoproliferative disorders. We recently published an account of changes in the gene expression profile during glucocorticoid-induced apoptosis in an acute lymphoblastic leukemia cell line treated for 2 and $8 \mathrm{~h}$ with dexamethasone. Although this study revealed a possible mechanism for glucocorticoid-induced apoptosis, the details of this hypothesis and whether it can be extended to lymphocytic leukemia cells from patients remain to be determined. Further analyses with DNA chips should help resolve this question and increase our understanding of the effects of glucocorticoids in acute lymphoblastic leukemia patients.

Toshihito, Nagata

\section{High level of $\mathrm{hCDC10}$ gene expression in neuroblastoma may be associated with favorable tumor characteristics}

Nagata Toshihito ${ }^{1}$, Takahashi Yasuo ${ }^{1}$, Asai Satoshi $^{1}$,

Mugishima Hideo ${ }^{2}$, Koshinaga Shigemichi ${ }^{3} \&$ Ishikawa Koichi $^{1}$

${ }^{1}$ Department of Pharmacology, Nihon University School of Medicine, Tokyo, Japan

${ }^{2}$ Department of Pediatrics, Nihon University School of Medicine, Tokyo, Japan

${ }^{3}$ Department of Pediatric Surgery, Nihon University School of Medicine, Tokyo, Japan

Clinical and biological heterogeneity within an individual tumor diagnostic category is derived from differences in messenger RNA profiles among tumor tissues, as well as differences in aberrations of genomic DNA, in many types of tumors. Neuroblastoma is a childhood tumor that shows clinical and biological hetero- geneity derived from several kinds of genetic and clinical variables. Age at diagnosis (under or over one year) has been reported to be the most important prognostic variable of neuroblastoma patients, and many reports have indicated that the biological behavior of neuroblastomas detected through mass screening (under one year of age) and that of mass-screening-negative, later-presenting neuroblastomas (over one year of age) is different. Using differential display and polymerase chain reaction with reverse transcription, we found that the mRNA for the human homologue of yeast CDC10 ( $h C D C 10)$ gene identified in Saccharomyces cerevisiae was differentially expressed in these two groups. A high level of $h C D C 10$ expression was associated with favorable clinical and biological characteristics of neuroblastoma tumors and a good prognosis. The expression of $h C D C 10 \mathrm{mRNA}$ in neuroblastoma tumors may affect the clinical and biological characteristics of this type of tumor.

Uchida, Kazuhiko

[48]

\section{Whole-chromosome imbalance pattern of comparative genomic hybridization in neuroblastomas is associated with regressive disease from mass screening cases showing spontaneous regression}

Kazuhiko Uchida ${ }^{1}$, Misako Hirai ${ }^{1}$, Sadao Yoshida $^{1}$, Hironobu Kashiwagi ${ }^{1}$, Saito Soichiro ${ }^{1}$, Michio Kaneko ${ }^{2}$, Ohkawa Haruo ${ }^{2}$, Akira Nakagawara ${ }^{3} \&$ Miwa Masanao ${ }^{1}$

${ }^{1}$ Institute of Basic Medical Sciences, University of Tsukuba, Tsukuba, Ibaraki 3058575, Japan

${ }^{2}$ Institute of Clinical Medicine, University of Tsukuba, Tsukuba, Ibaraki 305-

8575, Japan

${ }^{3}$ Biochemistry Division, Chiba Cancer Center, Chiba 260, Japan

Spontaneous regression of neuroblastoma occurs in infants (one year of age) at stage $4 \mathrm{~S}$, even those with metastases, and is observed in cases found by mass screening. Progression to fatal disease is most common in children over one year of age. The genetic heterogeneity of neuroblastoma has been established by comparative genomic hybridization. To clarify the differences in genetic backgrounds between regressive and progressive disease in neuroblastoma, we analyzed 45 cases using comparative genomic hybridization: 16 stage 4 cases with bone metastasis and 29 infant cases, including 7 stage $4 \mathrm{~S}$ cases and 22 mass-screening cases that were estimated to have good prognoses, with spontaneous regression and differentiation. Two distinct types of chromosomal imbalance patterns, whole-chromosome and regional changes, were observed in neuroblastomas. All 16 advanced cases showed multiple and regional gains and losses on comparative genomic hybridization profiles, as in other adult cancers. However, all spontaneously regressive cases in stage $4 \mathrm{~S}(6 / 7)$ and 18 of the 22 mass-screening cases showed whole-chromosome imbalances. Interestingly, all the patients with whole-chromosome patterns did not show characteristics known to be poor clinical and biological prognostic factors, and all have remained alive without evidence of disease. The whole-chromosome gains and losses may be due to aneuploidy rather than DNA amplification or deletion and may be involved in reversible tumorigenesis in neuroblastomas. These results imply that DNA copy number imbalance is a key step in carcinogenesis. 